\title{
Sample size calculation for clinical trials using cardiac magnetic resonance partition coefficient and extracellular volume fraction for the assessment of diffuse myocardial fibrosis
}

Songtao Liu ${ }^{1,2^{*}}$, Jing $\mathrm{Han}^{3}$, Marcelo Nacif ${ }^{1}$, Jacquin Jones ${ }^{1}$, Nadine Kawel ${ }^{1}$, Peter Kellman ${ }^{4}$, Christopher T Sibley ${ }^{1,2}$, David A Bluemke ${ }^{1,2}$

From 16th Annual SCMR Scientific Sessions

San Francisco, CA, USA. 31 January - 3 February 2013

\section{Background}

Cardiac magnetic resonance (CMR) T1 mapping has been used to characterize myocardial diffuse fibrosis. The aim of this study is to determine the reproducibility and sample size of CMR fibrosis measurements for use in clinical trials.

\section{Methods}

A modified Look-Locker with inversion recovery (MOLLI) sequence was used to determine myocardial T1 values pre-, and 12 and 25 min post-administration of a gadolinium-based contrast agent at 3 Tesla. For 24 healthy subjects ( 8 men; $29 \pm 6$ years), two separate scans were obtained a) with a bolus of $0.15 \mathrm{mmol} / \mathrm{kg}$ of gadopentate dimeglumine and b) $0.1 \mathrm{mmol} / \mathrm{kg}$ of gadobenate dimeglumine, respectively, with averaged of $51 \pm 34$ days between two scans. Separately, 25 heart failure subjects (12 men; $63 \pm 14$ years), were evaluated after a bolus of $0.15 \mathrm{mmol} / \mathrm{kg}$ of gadopentate dimeglumine. Myocardial partition coefficient $(\lambda)$ was calculated according to ( $\Delta \mathrm{R} 1$ myocardium $/ \Delta \mathrm{R} 1$ blood), and $\mathrm{ECV}$ was derived from $\lambda$ by adjusting (1-hematocrit).

\section{Results}

Mean ECV and $\lambda$ were both significantly higher in HF subjects than healthy (ECV: $0.287 \pm 0.034$ vs. 0.267 $\pm 0.028, \mathrm{p}=0.002 ; \lambda: 0.481 \pm 0.052$ vs. $0.442 \pm 0.037, \mathrm{p}<0.001$, respectively). For healthy subjects, the mean intra-study changes in ECV and $\lambda$ between 12 and 25 minutes were $0.007 \pm 0.006$ and $0.012 \pm 0.009$, respectively. The mean inter-study changes in ECV and $\lambda$ were $0.006 \pm 0.017$ and $0.016 \pm 0.025$, respectively. Thus, the inter-study ECV and $\lambda$ variation was about 2.8 times greater than the intra-study ECV and $\lambda$ variation in healthy subjects (ECV:0.017 vs. $0.006, \lambda: 0.025$ vs. 0.009 , respectively). In heart failure subjects, the intra-study differences between 12 and $25 \mathrm{~min}$ ECV and $\lambda$ were $0.007 \pm 0.017$ and $0.012 \pm 0.028$, respectively. The estimated sample size to detect a one standard deviation (SD) change of ECV $(0.035)$ or $\lambda(0.05)$ with a power of $80 \%$ and an alpha error of 0.05 for heart failure subjects using a two group design was 31 and 40 in each group, respectively.

\section{Conclusions}

ECV and $\lambda$ quantification have a low variability across scans, and could be a viable tool for evaluating clinical trial outcome. ECV requires a smaller sample size than $\lambda$ to detect group differences from treatment.

\section{Funding}

National Institutes of Health Intramural program.

${ }^{1}$ Radiology and Imaging Sciences, NIH Clinical Center, Bethesda, MD, USA Full list of author information is available at the end of the article 


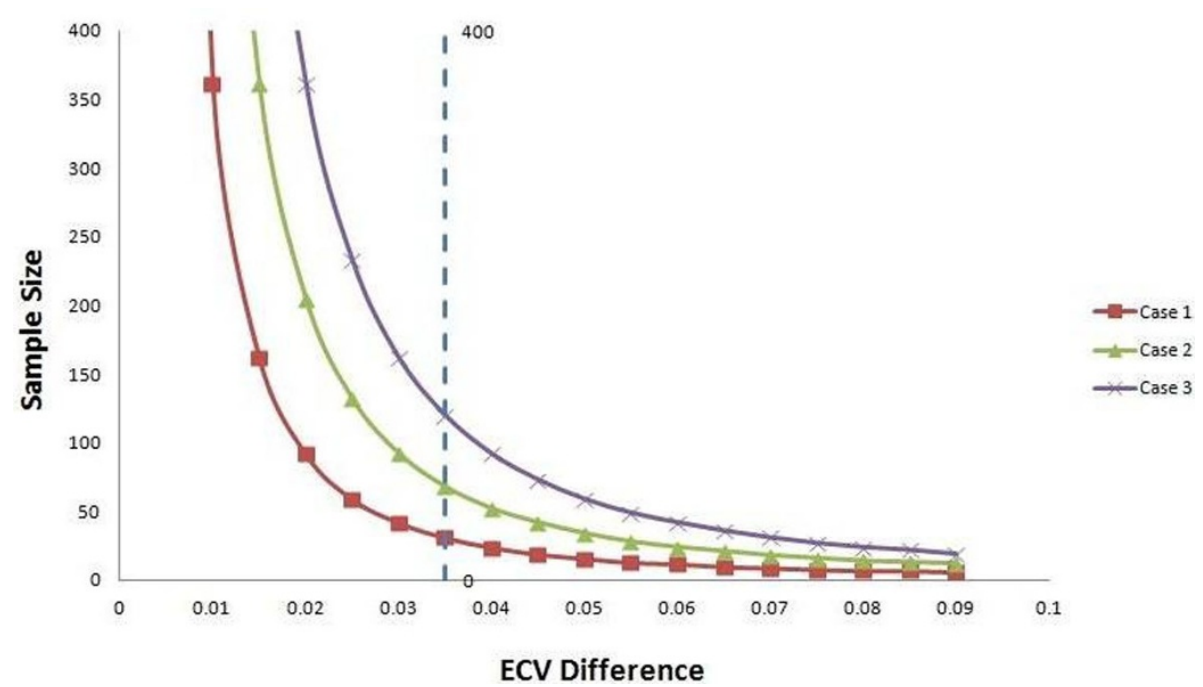

Figure 1 Sample size required in each group to detect a certain ECV difference with a two group design of 80\% power and an alpha error of 0.05. The $X$ axis values corresponding to the ECV difference need to be detected like the first column in Table 1. The three curves corresponding to case 1,2 and 3 of Table 1 .

Table 1 Estimated sample size in heart failure group to detect the change of ECV and $\lambda$ with a power of $80 \%$

\begin{tabular}{ccccccc}
\hline Clinical change & \multicolumn{2}{c}{ Case 1 } & \multicolumn{2}{c}{ Case 2 } & \multicolumn{2}{c}{ Case 3 } \\
\hline & SDD1 & N1 & SDD2 & N2 & SDD3 & N3 \\
\hline$\lambda(0.05)$ & 0.078 & 40 & 0.117 & 87 & 0.156 & 154 \\
ECV $(0.035)$ & 0.048 & 31 & 0.072 & 68 & 0.096 & 120 \\
\hline
\end{tabular}

Sample size need to detect a clinical meaning change of ECV and $\lambda$ with $80 \%$ of power and an alpha error of 0.05 . Sample size is derived from the interstudy SDD. Note that for studies comparing active vs. placebo, these sample size numbers need to be doubled. Case 1: the inter-study SDD1 in HF group was estimated 3 fold greater than the intra-study SDD; Case 2, the inter-study SDD2 was estimated 1.5 times more than SDD1; Case3, the inter-study SDD3 was estimated 2 times more than SDD1.

\section{Author details}

${ }^{1}$ Radiology and Imaging Sciences, NIH Clinical Center, Bethesda, MD, USA. ${ }^{2}$ Molecular Biomedical Imaging Laboratory, NIBIB, Bethesda, MD, USA. ${ }^{3}$ US FDA, Rockville, MD, USA. ${ }^{4}$ Laboratory of Cardiac Energetics, NHLBI, Bethesda, $M D$, USA.

Published: 30 January 2013

\section{doi:10.1186/1532-429X-15-S1-P39}

Cite this article as: Liu et al:: Sample size calculation for clinical trials using cardiac magnetic resonance partition coefficient and extracellular volume fraction for the assessment of diffuse myocardial fibrosis. Journal of Cardiovascular Magnetic Resonance 2013 15(Suppl 1):P39. 